ICONE19 - 44012

\title{
NUCLEAR POWER PLANT PERFORMANCE MONITORING USING DATA VALIDATION AND RECONCILIATION (DVR) - APPLICATION AT THE BRAZILIAN ANGRA 2 PWR PLANT
}

\author{
Anh Tho Tran Quang \\ BELSIM S.A., Rue de Bruxelles 174K, B-4340 Awans, Belgium \\ Email: anhtho.tranquang@ belsim.com Tel: +32 (0) 42598888 Fax: +32 (0) 42598889 \\ Edson Azola \\ ELETROBRAS Eletronuclear, Rua da Candelária, 65 Centro, 20091-906, Rio de Janeiro, Brazil \\ Email: eazola@eletronuclear.gov.br Tel: +55 (21) 25887615 \\ Hervé Closon \\ BELSIM S.A., Rue de Bruxelles 174K, B-4340 Awans, Belgium \\ Email: herve.closon@belsim.com Tel: +32 (0) 42598888 Fax: +32 (0) 42598889 \\ Robert Chares \\ BELSIM S.A., Rue de Bruxelles 174K, B-4340 Awans, Belgium \\ Email: robert.chares@belsim.com Tel: +32 (0) 42598888 Fax: +32 (0) 42598889
}

Keywords: Data validation and reconciliation (DVR), units performance monitoring and optimization, process inefficiencies detection, faulty sensors detection, maintenance strategy, heat and mass balances based on full thermodynamic package, acceptance tests conduction, reactor thermal power calculation.

\section{ABSTRACT}

Operational decisions related to plant performance monitoring mainly result from raw process measurement analysis. When first signs of sub-optimal behavior occur, traditional methods mainly focus on the observation of selective pieces of information. The main disadvantages of these methods are:

- Investigation efforts are required to localize the problems, entailing time losses and costs;

- Validity and reliability of the pieces of information cannot be checked as long as the measurements are observed individually.

The problem is not the lack of methods and techniques but rather a lack of reliable and consistent data and information across the entire plant.

To overcome drawbacks of traditional methods, measurements are considered as interacting with one another. When related to the other measurements of the plant, the observed information becomes of an interest: its incoherency to the others identifies and localizes a problem.

The Data Validation and Reconciliation technology (DVR) is based on an advanced process data coherency treatment. By using all available plant information and by closing the plant heat and mass balances based on rigorous thermodynamics, the method generates:

- A single set of reliable and most accurate plant process data;

- Alarms for incoherent measurements, highlighting potential problems;

- Alarms for equipment and process performance degradation;

- Alarms for faulty and drifting measurements.

The use of the advanced DVR software package VALI offers various benefits as it allows to base operational decisions on reliable and accurate data.

\section{Support for sub-optimal behaviors diagnostic}

By real-time reconciliation of the plant process measurements, the operators will be automatically warned of incoherent measurements of the plant, which pinpoints the roots of suboptimal and deviating plant behaviors. Emerging problems, either on the equipment, process or sensor level, are detected early with lowest efforts. 


\section{Performance optimization}

The reconciliation of plant process measurements gives direct insight into the plant operating conditions, including the computation of key performance indicators and missing data, with the highest level of precision.

It allows operators to

- Work closer to the limits without compromising safety;

- Conduct automatic acceptance tests before and after outage/retrofit/maintenance;

- Identify key parameters impacting the plant performance;

- Monitor performance of equipments and processes;

- Optimize sensor maintenances.

This paper presents an application of DVR techniques for performance monitoring, implemented at the Brazilian Angra 2 nuclear power plant - pressurized water reactor (PWR). It describes the benefits obtained until date (automatic detection of sensor drifting, equipment degradation, leakages, process changes, etc.) as well as additional uses of the application (acceptance tests, lost megawatts tracking, etc.).

\section{INTRODUCTION}

\subsection{Measurement errors}

In the process industry, all raw measurements are erroneous. The sources of errors are numerous: intrinsic sensors precision, installation problems, fluctuations of the physical phenomenon, etc. The use of raw measurements without correction leads to

- Mass and energy imbalances at equipment or plant level;

- Key Performance Indicators (KPIs) that are drastically biased by measurement errors.

Moreover, raw measurements are used for monitoring the plant, taking operational decisions or conducting acceptance tests. The measurements used should then be relevant.

A simple mass balance illustrates on one hand the risks of using raw process measurements and on the other hand the useful and trustworthy information that can be extracted out of them. A stream $\mathrm{A}$ is split into two streams $\mathrm{B}$ and $\mathrm{C}(\mathrm{A}=\mathrm{B}+\mathrm{C})$ while one flow rate measurement along with its uncertainty is available on each stream. The stream A is considered as the KPI to be monitored.

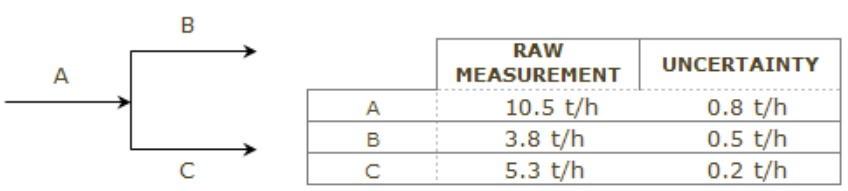

Figure 1: SIMPLE MASS BALANCE ILLUSTRATION

For monitoring the flow rate $\mathrm{A}$, if one considers that

- CASE 1: The flow rate measurement $B$ only is available: the system is undetermined. The measurement $\mathrm{A}$ is not accessible;

- CASE 2: The two flow rate measurements $B$ and $C$ only are available: the system is just determined. The flow rate $\mathrm{A}$ is deduced from the flow rate raw measurements $\mathrm{B}$ and $\mathrm{C}$ that are used as such $(\mathrm{A}=\mathrm{B}+$ $\mathrm{C}=9.1 \mathrm{t} / \mathrm{h})$;

- CASE 3: The three flow rate measurements $A, B$ and $C$ are simultaneously available: the system is redundant, namely over-determined. The use of raw measurements yields a mass imbalance $(\mathrm{A}-\mathrm{B}-\mathrm{C}=$ $1.4 \mathrm{t} / \mathrm{h} \neq 0)$. Corrections based on measurement uncertainties are brought to raw data $\mathrm{A}, \mathrm{B}$ and $\mathrm{C}$ under the condition of fulfilling the mass balance constraint.

\begin{tabular}{|c|c|c|c|c|c|}
\hline & \multirow{3}{*}{ A } & & & \\
\hline & & & \multirow{2}{*}{$\begin{array}{l}\mathrm{B} \\
3.8\end{array}$} & \multirow{2}{*}{$\begin{array}{l}\mathrm{C} \\
-\end{array}$} & \multirow{2}{*}{$\begin{array}{c}\text { Mass balance } \\
=\mathrm{A}-\mathrm{B}-\mathrm{C} \\
\text { Not applicable }\end{array}$} \\
\hline CASE 1 & Undetermined & & & & \\
\hline CASE 2 & Just determined & 9.1 & 3.8 & 5.3 & Not applicable \\
\hline \multirow{2}{*}{ CASE 3} & $\begin{array}{l}\text { Over determined } \\
\text { (measured) }\end{array}$ & 10.5 & 3.8 & 5.3 & 1.4 \\
\hline & $\begin{array}{l}\text { Over determined } \\
\text { (reconciled) }\end{array}$ & 9.54 & 4.18 & 5.36 & 0 \\
\hline
\end{tabular}

Table 1: Flow RATes A, B AND C (T/H) - Bold VAlues ARE RAW MEASUREMENTS; CASE 1: FLOW RATE A IS NOT ACCESSIBLE; CASE 2: FLOW RATE A IS DEDUCED FROM B AND C; CASE 3: FLOW RATES A, B, C ARE CORRECTED AND MASS BALANCE IS CLOSED.

The last case introduces how the interconnection of measurements with all of the available information can be exploited. Raw measurements are no longer used as individual data but as an interdependent network of information that allows the determination of the most probable state of the process.

The complexity increases when dealing with many variables connected by several constraints including mass and energy balances, where raw data corrections cannot be made manually.

\subsection{Data Validation and Reconciliation}

\section{THEORETICAL BACKGROUND}

Data Validation and Reconciliation (DVR) is a technique that targets at correcting measurement errors which are due to measurement noise, i.e. random errors (cf. [1] and [2]). From a 
statistical point of view, the main assumption is that no systematic errors exist in the set of measurements, since they may bias the reconciliation results and reduce the robustness of the reconciliation.

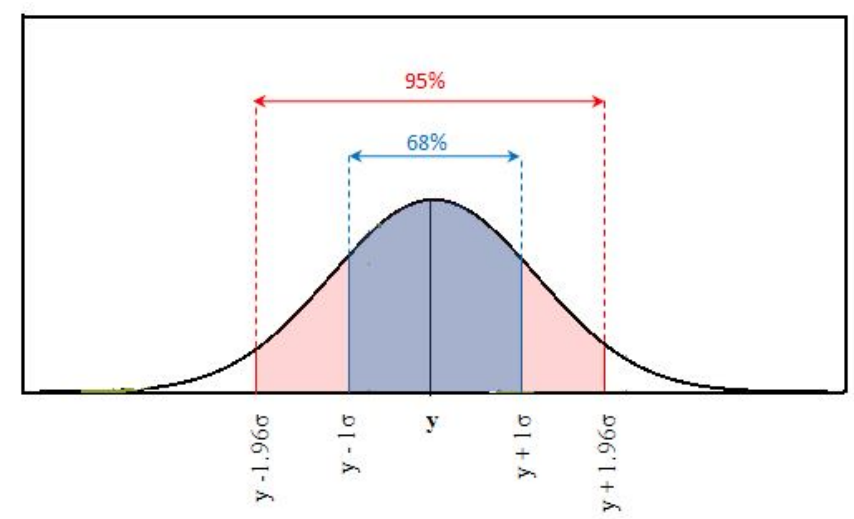

Figure 2: GAUSSIAN DISTRIBUTION OF MEASUREMENT ERRORS

VDI 2048 is a guideline approved by the Association of German Engineers (VDI) which describes how raw measurements in power plant should be corrected (cf. [3]). It essentially describes the technology of Data Validation and Reconciliation as it is presented in this article. DVR in accordance with VDI 2048, minimizes the correction brought to each raw measurement in the sense of least squares, such as the reconciled values become coherent to a set of constraints. Given $n$ measurements $y_{i}$ :

$$
\min _{x, y^{*}} \sum_{i=1}^{n}\left(\frac{y_{i}^{*}-y_{i}}{\sigma_{i}}\right)^{2}
$$

Subject to

$$
\begin{aligned}
& F\left(x, y^{*}\right)=0 \\
& y_{\min } \leq y^{*} \leq y_{\max } \\
& x_{\min } \leq x \leq x_{\max },
\end{aligned}
$$

where

$y_{i}^{*}$ is the reconciled value of the $i$-th measurement $(i=1, . ., n)$;

$\mathrm{y}_{\mathrm{i}}$ is the measured value of the $\mathrm{i}$-th measurement $(\mathrm{i}=1, \ldots, \mathrm{n})$;

$\sigma_{\mathrm{i}}$ is the standard deviation of the $\mathrm{i}$-th measurement $(\mathrm{i}=1, . ., \mathrm{n})$;

$\mathrm{x}_{\mathrm{j}}$ is the $\mathrm{j}$-th unmeasured variable $(\mathrm{j}=1, . ., \mathrm{m})$;

$\mathrm{F}\left(\mathrm{x}, \mathrm{y}^{*}\right)=0$ are the process equality constraints;

$\mathrm{x}_{\min }, \mathrm{x}_{\max }, \mathrm{y}_{\min }, \mathrm{y}_{\max }$ are the bounds on the measured and unmeasured variables;

$\left(\frac{y_{i}^{*}-y_{i}}{\sigma_{i}}\right)^{2}$

is called the penalty of measurement $\mathrm{i}$.
The objective function is the sum of penalties

$$
\sum_{i=1}^{n}\left(\frac{y_{i}^{*}-y_{i}}{\sigma_{i}}\right)^{2} \text {. }
$$

denoted by $\xi_{0}$.

It turns out that, under those assumptions and with a probability of $95 \%$, the absolute difference between the measured value and the true value is less than twice the standard deviation $(1.96 \sigma)$.

The constraints are set up by representing the plant process in a flow-sheet, where

- All available measurements along with their confidence intervals are implemented;

- Relations between the variables are based on mass and energy balances and on a full rigorous thermodynamic package;

- Technical data of equipments (rotating machine characteristic curves, heat transfer coefficient, etc.) and key performance indicators are incorporated;

- Automatic detection of different operating modes is handled.

\section{QUALITY CRITERIA}

To validate the reliability of the reconciled values, the DVR results are subject to a statistical criterion based on VDI 2048 consideration.

If there are no gross errors present in the set of measured values, then each penalty term in the objective function is a random variable that follows a standard normal distributed ( mean equal to 0 , variance equal to 1 ). Therefore, the objective function is a random variable which follows a chi-square distribution, since it is the sum of the square of normally distributed random variables. One obtains an indication of whether a gross error exists when comparing the value of the objective function $\xi_{0}$ with a given percentile $\chi^{2} \alpha$ of the probability density function of a chi-square distribution (e.g. the 95 th percentile for a $95 \%$ confidence). For example, if $\xi_{0} \leq \chi^{2} 95 \%$, then no gross errors exist with $95 \%$ probability.

The global criterion:

$$
\text { Quality }=\xi_{0} / \chi^{2} 95 \% .
$$

The Quality > 1 indicates significant suspicion that some measurement errors bias the DVR results. Different scenarios are possible: a biased flow meter, a leakage, etc.

The mass balance example related to figure 1 and table 1 CASE 3 shows a Quality > 1: 


\begin{tabular}{|l|cccc|}
\hline WEIGHTED SQUARE & A & B & C & SUM \\
OF ERRORS & 5.571 & 2.176 & 0.348 & 8.095 \\
\hline CHI SQUARE X S $^{2} \%$ & \multicolumn{5}{|c|}{3.84} \\
\hline QUALITY & \multicolumn{5}{|c|}{2.11} \\
\hline
\end{tabular}

Table 2: MASS BALANCE QuALITY DETAILS

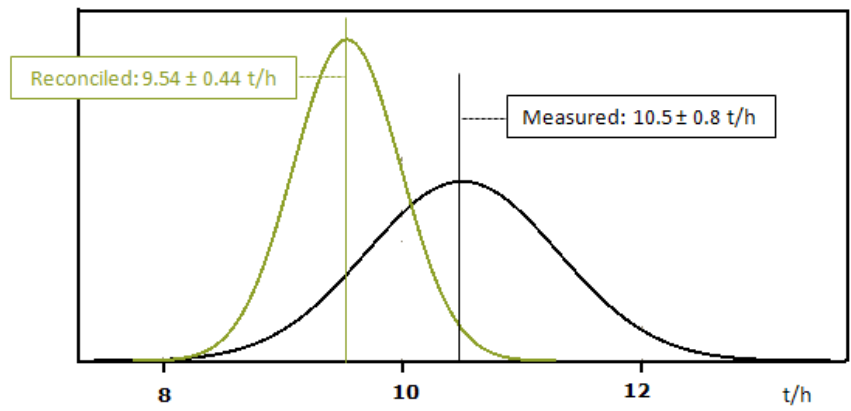

Figure 3: MASS BALANCE - FLOW RATE A MEASUREMENT ERROR

Gross errors are systematic measurement errors that may bias the reconciliation results. Therefore it is important to identify and eliminate these gross errors from the reconciliation process. Once a gross error has been detected, there are two major approaches how to deal with them:

- Gross error elimination;

- Gross error relaxation.

Gross error elimination determines one or more measurement that are biased by systematic errors and eliminates them sequentially or simultaneously from the data set. Once the gross errors are discarded from the measurements, the reconciliation can be done without these faulty measurements that perturb the quality of the reconciliation results. The elimination is then repeated until no indication is given that gross errors exist in the set of measurements.

Gross error relaxation means that the measurement uncertainty is increased until the reconciled value is within the $95 \%$ confidence interval. Relaxation typically is applied when it is not possible to determine which measurement is responsible for the gross error.

\section{INDUSTRIAL BENEFITS}

The main characteristics of the DVR technology is to provide an automatic access to a single set of reconciled data (coherent and reliable) - the most probable state of the plant, where

- All mass, energy and thermodynamic balances are fulfilled;

- All measurement uncertainties are reduced based on the computation of an embedded error propagation system;
- KPIs and unmeasured data are computed together with the lowest uncertainties, deduced from redundant measurements and their relationships.

Based on the theoretical considerations, the DVR technology benefits directly derive from the automatic highlight of suspicious measurements. DVR thus allows the minimization of time for detection of either faulty sensors or plant process inefficiencies or equipments degradation.

Other typical benefits of applying the technology of DVR to the nuclear power industry can be found at [4].

\section{DVR APPLICATION AT THE ANGRA 2 PWR PLANT}

The Angra 2 PWR plant has been depicted in a DVR model using VALI software. The model consists in primary, secondary and tertiary cycles. It includes 323 raw measurements (167 temperatures, 78 pressures, 41 flow rates, 16 pump rotating speeds, 14 binary indicators, 5 electrical powers and 2 valve stem positions). The level of redundancy is 178. The model scope is detailed in Appendix A. Automatic reconfiguration of the model has been implemented to support the different plant operating modes.

The DVR Application is running on line every 15 minutes, following a customized workflow:

- 15 minutes average measurements are acquired by the system from a data historian;

- Raw measurements are pre-validated by a filtering system to ensure that the inputs of the reconciliation make sense with regards to the operating modes;

- Reconciled data are computed based on the input and are stored in a dedicated database;

- Gross errors are detected and eliminated (if some exist);

- DVR results are retrieved from the database by means of a dedicated analysis tool.

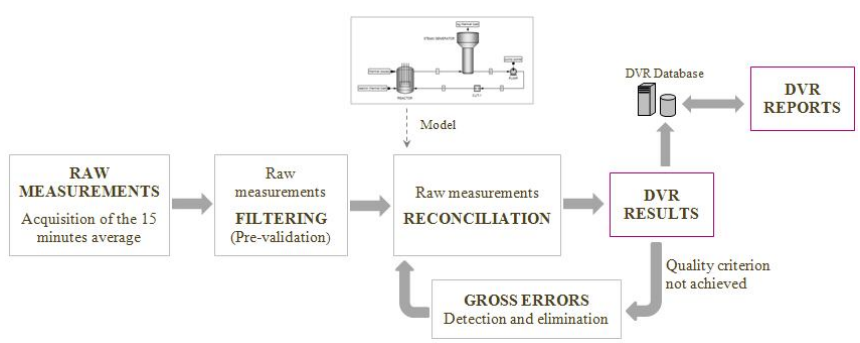

Figure 4: DVR APPLICATION WORKFLOW 
The benefits described in this paper have been pinpointed by the DVR results analysis.

\subsection{Heat and mass balances report}

Assessments of Performance Acceptance Test based on raw process measurement data usually yields incoherence in the balances (mass, energy and thermodynamic). Traditional methods for closing balances require resources and workload. Moreover these methods are most often based on experiences and the results depend on human expertise.

The DVR Application has been used as a tool for on line heat and mass balances assessments based on reconciled available measurement process data, without requiring additional instrumentation or time consuming manual corrections.

The heat and mass balances of the complete primary and secondary cycles are generated every 15 minutes, which gives access to

- A clear and reliable picture of the complete heat cycles;

- The performance of acceptance tests before and after plant outage, retrofit or maintenance.

The heat and mass balance report has been fully customized based on the plant standards and unmeasured KPIs have been added on request.

\subsection{Reactor thermal power}

Prior to VALI installation, a dedicated tool using raw measurements was used for reactor thermal power computation. The DVR Application has now enabled online access to the reactor thermal power with an accuracy of $0.38 \%$.

The reactor thermal power calculated by VALI has indicated that the active electric power could be increased up to 5 $\mathrm{MW}_{\text {electrical }}$ without compromising safety. The electric power gain has been verified by a comparison of the reactor thermal power computed by the plant tool and VALI.

The operational personnel has changed its procedure to use the reactor thermal power computed by the DVR Application.

\subsection{Average temperature}

Up to now, a dedicated procedure and additional measurements were implemented for the calculation of primary cycle average temperatures, used for calibration. A stratification phenomenon has been observed due to the sensors localized all around the pipes.

The procedure is now implemented in the DVR Application allowing an online access to the average temperature with the highest level of accuracy. The stratification phenomenon has been drastically reduced and the reconciled average temperature can be used as a reference for calibration.

\subsection{Condition based maintenance}

While systematic and preventive maintenance requires continuous attention and inspections, VALI condition-based maintenance rises alarms right at the moment when a problem occurs, pinpointing its localization.

The DVR Application has enabled early detection of faulty and drifting sensors in the whole plant.

Amongst the suspicious sensors detected by VALI, the condenser outlet pressures have been identified as biased. On site investigation has confirmed the finding. Figure 5 illustrates the measured and reconciled values of the outlet pressure of one of the three condensers.

\section{CONDENSER OUTLET PRESSSURE - BARABS}
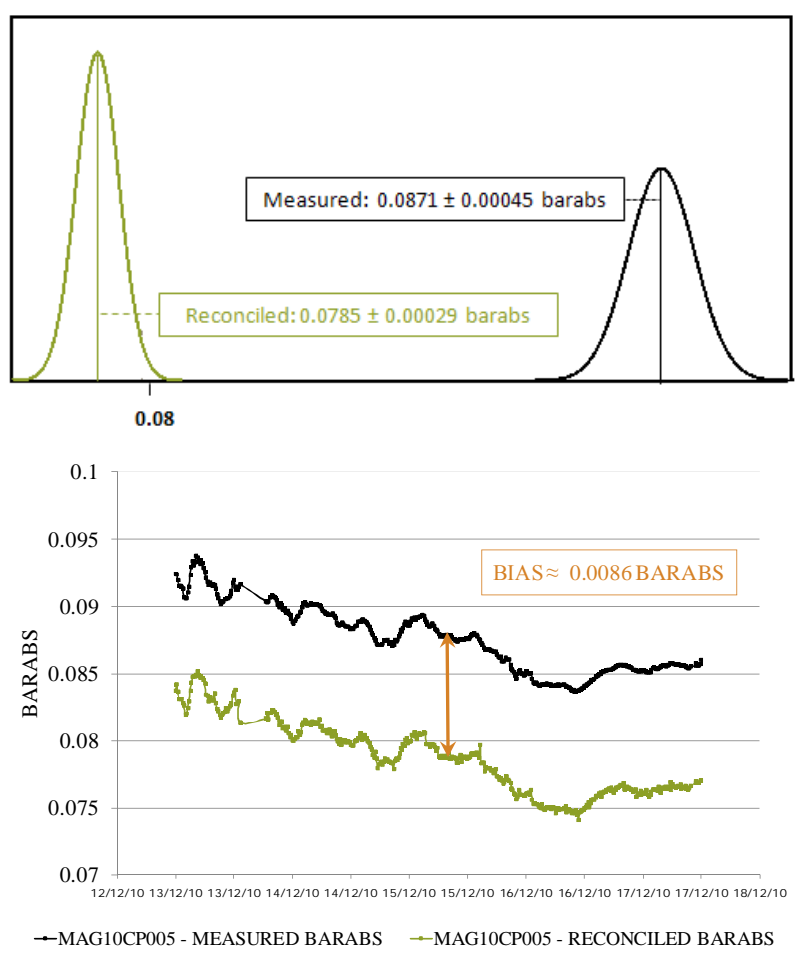

Figure 5: CONDENSER OUTLET PRESSURE - DRIFTING MEASUREMENT

While the condenser outlet pressure is a key parameter for performance monitoring of nuclear power plants, a sensitivity analysis has shown that a bias of 0.0086 bar on each condenser has an impact on the reactor thermal power of $2.1 \mathrm{MW}_{\text {thermal. }}$.

A list of faulty and drifting sensors, automatically generated by VALI, has allowed the operators to target their maintenance. Reconciled data can be used as calibration references. 


\subsection{Performance monitoring}

Generally, time elapses between the first emergence of a suboptimal behavior and the operator's awareness of the problem and his/her ability to localize the process inefficiencies.

The DVR Application has allowed the operators to optimize their performance thanks to an automatic and early detection of equipment degradation and process inefficiencies. KPIs related to all equipments (heat exchangers, pumps, turbines and tanks) for the monitoring of their performance have been implemented.

\section{EQUIPMENTS DEGRADATION}

The access to equipment specific KPIs with highest level of accuracy and to a clear and reliable picture of the plant, has allowed the detection of a mass and energy imbalances around the HP reheaters A6. On site investigation has enabled to detect a leakage on one of the two reheaters A6. The leakage has been partially fixed during plant outage. Meanwhile the equipment complete fixing, the DVR model was adjusted accordingly with the modeling of an adequate leakage.

Figure 6 illustrates the reheaters A6 loads computation on the two identical lines 1 and 2, after model adjustment. A suboptimal behavior is observed on the HP reheater A6 line 1.

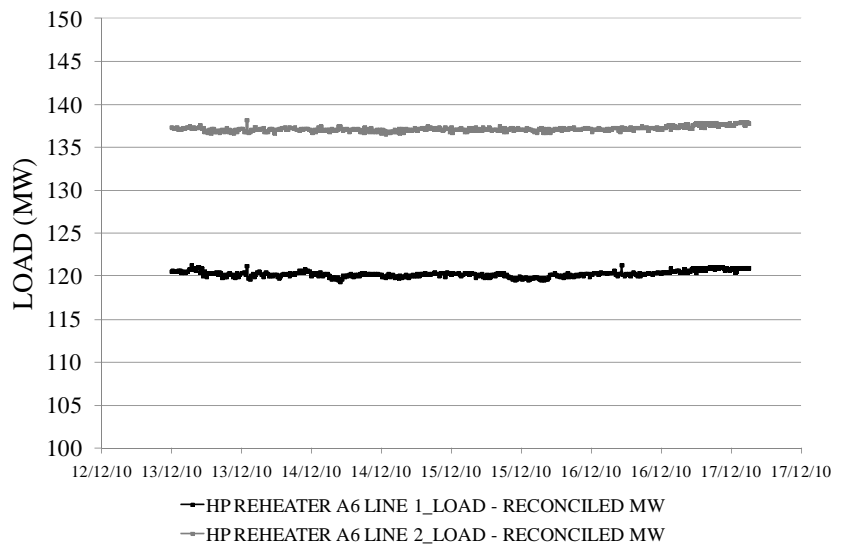

Figure 6: HP REHEATERS A6 LOAD COMPUTATION

\section{PROCESS INEFFICIENCIES}

The DVR results analysis has also enabled to localize process inefficiency around a recycle flow. The recycle flow returning back to the main feed water tank was expected to be around 8 $\mathrm{kg} / \mathrm{s}$ based on design. On site investigation has demonstrated a recycle flow of about $50 \mathrm{~kg} / \mathrm{s}$, which has been explained by the destruction of 4 out of 5 flow restrictions.

Such a recycle flow has direct impact on

- The consumption of the main feed water pumps (increase of approximately $0.5 \mathrm{MW}_{\text {electrical }}$ );

- The thermal power production and the energy efficiency of the secondary loop.
The flow restrictions have been fixed during plant outage

\section{CONCLUSION}

The DVR Application is a tool that addresses multiple challenges arising in the nuclear power industry, such as:

- Equipment monitoring;

- Tracking of plant inefficiencies;

- Condition based maintenance.

The operational personnel have access to an online and automated visualization of the true performance of the plant, by means of a set of reliable and coherent data with lowest level of uncertainty. Operational decisions can now be based on a clear and reliable image of the plant process data.

All reconciled KPIs are automatically generated, along with a quality certificate, enabling the performance monitoring at any level (plant, equipments, etc.). Alarms warn the process engineers when sub-optimal behaviors, either equipment degradations or plant inefficiencies, are detected. Plant and equipment performance is optimized thanks to an early detection of problems.

Condition-based maintenance is only effective when based on relevant and highly accurate information. VALI gives access to this information and automatically provides a list of suspicious sensors, either faulty or drifting.

The benefits of the DVR Application also extend to safety concerns such as reactor thermal power and primary average temperature, which are now computed based on reconciled data with the lowest level of uncertainty.

There were three important keys for the success of this project. First, there was a strong commitment to this project of the management of the Angra 2 nuclear plant and a positive attitude towards a management of change which ensured a permanent support from the nuclear power plant. Second, a dedicated multi-disciplinary team was established, formed by members from the Angra 2 plant and from Belsim, working together towards the defined objectives. Third, a transfer of knowledge was provided between Belsim and the Angra 2 plant personnel by various trainings, on-site missions and continuous support. These factors not only guaranteed that the project was successful for both parties, but also ensured a sustainable application and its continuous improvement even after the project has been completed. 


\section{ACKNOWLEDGEMENTS}

The authors would like to thank Professor Georges Heyen and Bruno Vrielynck for kindly reading early drafts of this paper and for giving useful comments that improve the quality of this paper.

\section{REFERENCES}

[1] P. Joris, B. Kalitventzeff, Process measurements analysis and validation, Proc. CEF'87: Use Comput. Chem. Eng., Italy, 41-46, 1987.

[2] Th. Amand, G. Heyen, B. Kalitventzeff, Plant Monitoring and Fault Detection: Synergy between Data Reconciliation and Principal Component Analysis, Comp. and Chem, Eng. 25, p. 501-507, 2001.

[3] VDI-Gesellschaft Energie und Umwelt, VDI Guidelines 2048 - Blatt 1, Uncertainties of measurements at acceptance tests for energy conversion and power plants - Fundamentals, www.vdi.de, 2000.

[4] M. Langenstein, J. Jansky, B. Laipple, Finding Megawatts in nuclear power plants with process data validation, Proceedings of ICONE12, Arlington, USA, April 25-29, 2004.

\section{APPENDIX A}

The DVR model consists of

- Primary cycle: Reactor vessel, steam generators, reactor coolant pumps, pressurizer;

- Secondary cycle: Steam generators, high and low pressure turbines including extraction, drain and sealing systems and alternator, moisture separators and reheaters, moisture separator condensate pumps, condensers, main condensate pumps, gland steam condenser, low pressure reheaters, low pressure preheater condensate pumps, main feed water tank, main feed water pumps, high pressure reheaters, steam trap system, auxiliary steam system (auxiliary steam condensate coolers, auxiliary steam condensate pumps), clean drain tanks, clean drain pumps, blowdown system (blowdown flash tanks, blowdown coolers);

- Tertiary cycle: condensers and sea water pumps. 